werden sich hieran schnell grundsätzlich staatsrechtliche Folgefragen anschließen, deren Wirkung im gesamten öffentlichen Recht zu spüren sein werden. So darf man gespannt sein, unter welchen Voraussetzungen die höchsten Gerichte etwa von einem gesetzgeberischen Normierungsversagen ausgehen und welche Rechtsfolgen sie hieran knüpfen werden.

Open Access. Dieser Artikel wird unter der Creative Commons Namensnennung 4.0 International Lizenz veröffentlicht, welche die Nutzung, Vervielfältigung, Bearbeitung, Verbreitung und Wiedergabe in jeglichem Medium und Format erlaubt, sofern Sie den/die ursprünglichen Autor(en) und die Quelle ordnungsgemäß nennen, einen Link zur Creative Commons Lizenz beifügen und angeben, ob Änderungen vorgenommen wurden.

Die in diesem Artikel enthaltenen Bilder und sonstiges Drittmaterial unterliegen ebenfalls der genannten Creative Commons Lizenz, sofern sich aus der Abbildungslegende nichts anderes ergibt. Sofern das betreffende Material nicht unter der genannten Creative Commons Lizenz steht und die betreffende Handlung nicht nach gesetzlichen Vorschriften erlaubt ist, ist für die oben aufgeführten Weiterverwendungen des Materials die Einwilligung des jeweiligen Rechteinhabers einzuholen.

Weitere Details zur Lizenz entnehmen Sie bitte der Lizenzinformation auf http://creativecommons.org/licenses/by/4.0/deed.de.

Open access funding provided by Projekt DEAL.

https://doi.org/10.1007/s10357-020-3692-3

\title{
Das Klimaschutzgesetz des Bundes - Hintergrund, Regelungsstruktur und wesentliche Inhalte
}

\author{
Juliane Albrecht*
}

C Der/die Autor(en) 2020

Das im Dezember 2019 in Kraft getretene Bundes-Klimaschutzgesetz (KSG) setzt in Deutschland erstmals einen dauerhaft verbindlichen Rahmen für den Klimaschutz und knüpft insofern an völker- und europarechtlichen Verpflichtungen an. Dieser Beitrag beschreibt den rechtlichen Hintergrund, die Regelungsstruktur und wesentliche Inhalte des Gesetzes. Dabei werden die einzelnen Vorschriften des KSG erläutert und mit den internationalen Vorgaben (Pariser Abkommen) sowie den aktuellen Instrumenten der EUKlimaschutzpolitik (Emissionshandels-RL, Klimaschutz-Verordnung, LULUCF-Verordnung und Governance-Verordnung) in Beziehung gesetzt sowie einer zusammenfassenden Bewertung unterzogen.

\section{Einführung}

Klimaschutz ist bereits seit längerem in der politischen und rechtlichen Diskussion. In etwa der Hälfte der Bundeländer liegen mittlerweile Klimaschutzgesetze vor. Seit dem Abkommen von Paris und „Fridays for Future“ ist das Thema mittlerweile auch auf Bundesebene ganz oben auf die politische Agenda gerückt. So hat die Bundesregierung im November 2016 den Klimaschutzplan 2050 als Langfriststrategie zum Pariser Übereinkommen vorgelegt. Am 18. Dezember 2019 ist nunmehr das Klimaschutzgesetz (KSG) in Kraft getreten. ${ }^{1}$ Damit wurde in Deutschland auf Bundesebene erstmals ein Klimaschutzgesetz mit verbindlichen Klimaschutzzielen eingeführt. Ziel des vorliegenden Beitrages ist es, Hintergrund, Regelungsstruktur die wesentlichen Inhalte des Gesetzes vorzustellen. Da die Vorgaben des KSG maßgeblich durch völker- und europarechtliche Anforderungen bestimmt sind, werden diese zunächst unter 2. näher beschrieben. Kap. 3 befasst sich mit der Entstehungsgeschichte des KSG, dessen Regelungsstruktur und wesentlichen Vorschriften. Der Beitrag schließt mit einer zusammenfassenden Bewertung der gesetzlichen Neuregelungen (Kap. 4).

Dr. Juliane Albrecht,

Leibniz-Institut für ökologische Raumentwicklung,

Dresden, Deutschland

\section{Völker- und europarechtliche Zusammenhänge}

Die Inhalte des neuen Bundesklimaschutzgesetzes erschließen sich maßgeblich vor dem Hintergrund völker- und europarechtlicher Vorgaben.

\subsection{Völkerrecht}

Grundlage des KSG ist das Übereinkommen von Paris (Paris Agreement, PA 2015)², welches aufgrund der Klimarahmenkonvention der Vereinten Nationen (UNFCCC, 1992) ${ }^{3}$, abgeschlossen worden ist und das Kyoto-Protokoll ${ }^{4}$ ablöst. Die vormals schematische Zweiteilung in Industrieländer einerseits und Schwellen- und Entwicklungsländer andererseits mündet in einem multilateralen Klimaabkommen, welches sämtliche Vertragsstaaten, freilich unter Berücksichtigung ihrer Leistungsfähigkeit, verpflichtet. ${ }^{5}$ Art. 2 Abs. 1a PA enthält die Verpflichtung, den Anstieg der globalen Durchschnittstemperatur auf deutlich unter 2 Grad und möglichst auf 1,5 Grad gegenüber dem vorindustriellen Niveau zu begrenzen, um die Auswirkungen des weltweiten Klimawandels so gering wie möglich zu halten. Das Abkommen sieht kein genau definiertes Zeitziel für die Klimaschutzanstrengungen vor, sondern ist darauf ausgerichtet, in der zweiten Hälfte dieses Jahrhunderts ein Gleichgewicht zwischen den anthropogenen Emissionen von Treibhausgasen aus Quel-

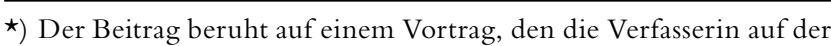
4. Bundesfachtagung Naturschutzrecht des Verbands Beruflicher Naturschutz (BBN) „Naturschutzrecht im Kontext von Klimawandel und Energiewende“ am 26.9.2019 an der Universität Kassel gehalten hat.

1) Bundes-Klimaschutzgesetz vom 12.12.2019 (BGB1. I S. 2513).

2) Paris Agreement, UNFCCC, Home, FCC/CP/2015/L.9/Rev.1 sowie BGBl. II 2016, 1082.

3) UN Framework Convention on Climate Change (UNFCCC), BGBl.1993 II 1784.

4) Kyoto Protocol to the United Nations Framework Convention on Climate Change, 1998; BGB1. II 2002, 966.

5) Saurer, NVwZ 2017, S. 1574; Schafhausen, in: Hebeler/Hofmann/ Proelß/Reif (Hrsg.), Die Zukunft der Energiewende, 2018 , S. 11, 20. 
len und dem Abbau solcher Gase durch Senken herzustellen (Art. 4 Abs. 1 PA). Um dieses Ziel zu erreichen, sind alle Staaten verpflichtet, nationale Klimaschutzbeiträge (,nationally determined contributions“, „NDCs“) zu erarbeiten und innerstaatliche Minderungsmaßnahmen zu ergreifen, um die Ziele dieser Beiträge zu verwirklichen (Art. 4 Abs. 2 PA). ${ }^{6}$ Die nationalen Klimaschutzziele werden dabei von den Staaten selbst festgelegt. ${ }^{7}$ Dieser ,,bottom-up“-Ansatz nimmt die Vertragsstaaten stärker in die Verantwortung und bindet sie intensiv in das internationale Regime ein. Für den Weg bis zum Erreichen der $\mathrm{CO}_{2}-\mathrm{Neutralität} \mathrm{regelt}$ das Abkommen einen Kommunikations- und Kontrollmechanismus, der eine weltweite Bestandsaufnahme vorsieht und die Mitgliedstaaten ggf. zum inhaltlichen Nachsteuern anhält (vgl. Art. $14 \mathrm{PA}) .{ }^{9}$ Alle fünf Jahre ist der nationale Klimaschutzbeitrag neu zu bestimmen (Art. 4 Abs. 9 PA) In diesem Rahmen müssen die Mitgliedstaaten über neue, ambitioniertere Ziele entscheiden (sog. Progressionsgebot, vgl. Art. 4 Abs. 3 PA).$^{10}$ Sowohl die EU als auch Deutschland haben das Abkommen ratifiziert. ${ }^{11}$

\subsection{Europarecht}

In ihrem Beitrag zum Übereinkommen von Paris (,NDC“, vgl. Art. 4 Abs. 16 PA) hat sich die EU verpflichtet, ihre THG-Emissionen bis 2030 um mindestens 40 Prozent gegenüber $1990 \mathrm{zu}$ senken. ${ }^{12}$ Die EU-Mitgliedstaaten hinterlegen zusätzlich keine eigenen nationalen Beiträge zum Paris-Abkommen, sondern wirken gemeinsam auf die Erfüllung des EU-Beitrags hin. ${ }^{13}$ Die EU-Kommission hat Ende 2018 die Vision eines Übergangs zur Treibhausgasneutralität bis 2050 vorgelegt. ${ }^{14}$ Diese wird nun im Rat der EU und im EU-Parlament diskutiert, um gemeinsam im Jahr 2020 eine langfristige Klimaschutzstrategie zum Übereinkommen von Paris zu hinterlegen (s. zu dieser Verpflichtung Art. 4 Abs. 19 PA)..$^{15}$ Deutschland hat mit dem im Jahr 2016 verabschiedeten Klimaschutzplan 2050 bereits eine solche Strategie formuliert. ${ }^{16}$ Zur Umsetzung der Klimaschutzziele stehen der EU die in Art. 288 AEUV genannten Handlungsformen zur Verfügung. Hiervon hat die EU durch verschiedene Rechtsakte Gebrauch gemacht. Der Beitritt der EU zum Pariser Übereinkommen untermauert somit den völkerrechtlichen Vertrag mit dem „robusten Rechtsdurchsetzungsinstrumentarium des Europarechts" (Vertragsverletzungsverfahren und Rechtskontrolle durch den EuGH). ${ }^{17}$ Eine zentrale Rolle spielen dabei die Richtlinien zum Emissionshandel, die Klimaschutzverordnung, die LULUCF-Verordnung sowie die GovernanceVerordnung, die im Folgenden näher betrachtet werden: ${ }^{18}$

\subsubsection{EU-Emissionszertifikatehandel}

Das zentrale europäische Instrument zur Reduktion von Treibhausgasemissionen in den Sektoren Energie und energieintensive Industrie ist der europäische Emissionszertifikatehandel. Er wurde 2005 zur Umsetzung des internationalen Klimaschutzabkommens von Kyoto eingeführt. Rechtliche Grundlage ist die Emissionshandelsrichtlinie (RL 2003/87/EG) ${ }^{19}$ (EHRL), welche durch die Richtlinien 2009/29/EG ${ }^{20}$ und 2018/410/EU ${ }^{21}$ geändert worden ist. Die Emissionshandelsrichtlinie zielt darauf ab, durch ein Emissionshandelssystem auf möglichst kosteneffiziente und wirtschaftlich verträgliche Weise eine Reduzierung der Treibhausgasemissionen zu erreichen (Art. 1). Durch das System wird eine Emissionsobergrenze (sog. Cap) für energieintensive Unternehmen in den o.g. Sektoren festgelegt. Diese Unternehmen dürfen nur die Emissionen ausstoßen, für die sie Emissionszertifikate besitzen. Die Zertifikate können auf dem Markt frei gehandelt werden. Hierdurch bildet sich ein Preis für den Ausstoß von Treibhausgasen und es entsteht ein wirtschaftlicher Anreiz zur Emissionseinsparung. ${ }^{22}$ Seit der dritten Handelsperiode (2013 bis 2020) erfolgt die Zuteilung nicht mehr über nationale Allokationspläne, sondern die EU-Kommission gibt eine EU-weite Gesamtobergrenze für $\mathrm{CO}_{2}$-Emissionen vor (vgl. Art. $9 \mathrm{EHRL}$ ). Allerdings existiert seit geraumer Zeit ein Überschuss an Zertifikaten, welcher zu niedrigen Preisen am Zertifikatemarkt geführt hat. ${ }^{23}$ Um Angebot und Nachfrage kurzfristig ins Gleichgewicht bringen, hatte die EU-Kommission 2014 durch eine Änderung der EU-EHS-Versteigerungsverordnung ${ }^{24}$ die Versteigerung von 900 Millionen Emissionszertifikaten aus dem Zeitraum 2014 bis 2016 auf 2019-2020 verschoben (sog. Backloading). ${ }^{25}$ Zudem wurde durch Beschluss (EU) 2015/1814 eine sog. Marktstabilitätsreserve (MSR) eingeführt, die ab dem Jahr 2019 jährlich 12\% der für die Versteigerung vorgesehenen Zertifikate zurückbehalten sollte, um ein Überangebot zu vermeiden. ${ }^{26}$ Mit dem Inkrafttreten der Richtlinie 2018/410/EU soll die Gesamtmenge an Zertifikaten für die vierte Handelsperiode (2021 bis 2030) weiter gesenkt werden. Als eine wesentliche Änderung ist eine Erhöhung des linearen Reduktionsfaktors im Hinblick auf die Emissionshöchstmenge von 1,74\% auf 2,2\% vorgesehen (Art. 9 Abs. 2 EHRL). Zudem wurde die Marktstabilitätsreserve für den Zeitraum bis 2023 auf 24 Prozent verdoppelt (später beträgt diese 12 Prozent). ${ }^{27}$ Sofern nicht anders beschlossen, verlieren in der Reserve befindliche Zertifikate, die über der Gesamtzahl der im vorangegangenen Jahr ver-

6) Näher hierzu Hofmann, in: Hebeler/Hofmann/Proelß/Reif (Hrsg.), Die Zukunft der Energiewende, 2018, S. 49, 52.

7) BMU, Die Klimakonferenz von Paris; https://www.bmu.de/ themen/klima-energie/klimaschutz/internationale-klimapolitik/ pariser-abkommen/ (Zugriff: 16.2.2020).

8) Schafhausen (Fn. 5), S. 20

9) Saurer (Fn. 5), 1575.

10) BMU (Fn. 7).

11) Durch Deutschland erfolgte die Ratifikation am 5.10.2016.

12) Submission by Latvia and the European Commission on Behalf of the European Union and its Member States, Intended Nationally Determined Contribution of the EU and its Member States, Riga, 6 March 2015

13) BMU, Klima- und Energiepolitik der Europäischen Union; https://www.bmu.de/themen/klima-energie/klimaschutz/euklimapolitik/ (Zugriff: 16.2.2020).

14) Europäische Kommission, Mitteilung der Kommission vom 28.11.2018. Ein sauberer Planet für alle, COM(2018) 773 final.

15) Näher hierzu: European Commission, 2050 Long Term Strategy, https://ec.europa.eu/energy/en/topics/energy-strategy-andenergy-union/2050-long-term-strategy.

16) BMU (Hrsg.), Klimaschutzplan 2050. Klimapolitische Grundsätze und Ziele der Bundesregierung, November 2016; näher hierzu Hofmann (Fn. 6), S. 49.

17) Saurer (Fn. 5), 1577; Hofmann (Fn. 6), S. 49 ff.

18) Weitere Rechtsakte mit Bezug zum Klimaschutz sind die Energieeffizienz-RL 2012/27/EU, ABl. L 315, S. 1 und die Erneuerbare-Energien-Richtlinie (2018/2001/EU), ABl. EU, L 328 , S. 82.

19) ABl. L 275, 25.10.2003, S. 32-46.

20) ABl. L 140, 5.6.2009, S. 63-87.

21) ABl. L 76, 19.3.2018, S. 3-27.

22) UBA, Der Europäische Emissionshandel, https://www.umweltbundesamt.de/daten/klima/der-europaeische-emissionshandel \#textpart-1 (Zugriff: 16-2-2020).

23) Vollmer, NuR 2018, 365. Als Gründe werden wenig ambitionierte Caps, krisenbedingte Produktions- und Emissionsrückgänge und die umfangreiche Nutzung von internationalen Projektgutschriften genannt, vgl. UBA (Fn. 22).

24) Verordnung (EU) Nr. 176/2014 der Kommission vom 25.2.2014, ABl. EU, L 56, 26.2.2014, S. 11.

25) Europäische Kommission, Strukturelle Reform des EU-Emissionshandelssystems, https://ec.europa.eu/clima/policies/ets/ reform_de (Zugriff: 16.2.2020).

26) Beschluss (EU) 2015/1814, ABl. EU, L 264, S. 1. In die Reserve wurden auch die 900 Mio. Zertifikate überführt, die im Rahmen des Backloading dem Markt bereits entnommen wurden, ebenso wie übriggebliebene Zertifikate der aktuellen Handelsperiode, vgl. Art. 1 Abs. 2 und 3 des Beschlusses (EU) 2015/1814

27) Vgl. Art. 2 Abs. 1 der Richtlinie 2018/410/EU. 
steigerten Zertifikate liegen, ab dem Jahr 2023 ihre Gültigkeit. ${ }^{28}$ Hierdurch soll bewirkt werden, dass auch künftig ein echter Minderungsanreiz besteht. ${ }^{29}$

\subsubsection{Klimaschutzverordnung}

Die Verordnung (EU) Nr. 2018/842 (Klimaschutzverordnung, auch „Lastenteilungsverordnung“, Effort-SharingRegulation) ${ }^{30}$ betrifft die Sektoren außerhalb des Emissionszertifikatehandels (Verkehr, Gebäude, Landwirtschaft und kleine Industrie-Anlagen). Diese Sektoren sind für fast $60 \%$ der Gesamtemissionen der EU verantwortlich. ${ }^{31}$ Im Gegensatz zu den Sektoren des EU-weiten Emissionshandels sind hier die Mitgliedstaaten für nationale Strategien und Maßnahmen zur Begrenzung der Emissionen verantwortlich. ${ }^{32}$ Um die erforderliche Emissionsreduktion $\mathrm{zu}$ erreichen bricht die Klimaschutzverordnung das Ziel der 30-prozentigen Emissionsreduktionen der gesamten EU für den Zeitraum bis 2030 gegenüber 2005 auf differenzierte Weise auf die einzelnen Mitgliedstaaten herunter. ${ }^{33}$ Die Minderungsanstrengungen betragen danach je nach Wirtschaftskraft der Mitgliedstaaten zwischen null und 40 Prozent gegenüber 2005 (vgl. Anhang I Klimaschutz-VO). Deutschland soll in den betroffenen Sektoren eine Treibhausgasreduktion in Höhe von 38 Prozent bis zum Jahr 2030 erreichen. Die Klimaschutz-VO enthält zudem Berechnungsregeln für den Pfad der jährlichen Emissionszuweisungen für die Jahre von 2021 bis 2029 (vgl. Art. 4). Die Emissionszuweisungen werden nicht auf einzelne Sektoren verteilt, allerdings sollen alle Sektoren zur Zielverwirklichung beitragen. ${ }^{34}$ Die nationalen Zuweisungen ergeben sich aus einem linearen Minderungspfad, der an einem Startpunkt im Jahr 2021 beginnt und bei einem fest definierten Zielwert für 2030 endet. $^{35}$ Demnach muss für jede emittierte Tonne $\mathrm{CO}_{2}$ eine Emissionszuweisung (,,annual emission allocation") aus dem gleichen Jahr (oder ggf. aus früheren Jahren) nachgewiesen werden (vgl. Art. 9 Klimaschutz-VO). ${ }^{36}$ Bei einer Überschreitung des nationalen Budgets muss der EU-Mitgliedstaat das Defizit ausgleichen. Dies ist möglich, indem z.B. Emissionszuweisungen von anderen EU-Mitgliedstaaten erworben werden (vgl. Art. 5 Abs. 5 Klimaschutz-VO). ${ }^{37}$ Gemäß der Verordnung (EU) Nr. 525/2013 $3^{38}$ sind die Mitgliedstaaten verpflichtet, jedes Jahr über ihre jeweiligen Treibhausgasemissionen zu berichten. Reichen die Fortschritte nicht aus, so muss der jeweilige Staat innerhalb von drei Monaten einen Plan mit Abhilfemaßnahmen vorlegen. Dieser umfasst zusätzliche nationale Politiken und Maßnahmen und einen strikten Zeitplan zur Durchführung gegenüber der Kommission (vgl. Art. 8 Klimaschutz-VO). ${ }^{39}$ Vorgängerinstrument zur Klimaschutzverordnung war die sog. Lastenteilungsentscheidung (Effort-Sharing-Decision, ESD, Entscheidung Nr. 406/2009/EG) ${ }^{40}$, die für Deutschland ein THG-Minderungsziel von 14\% bis 2020 im Vergleich zu 2005 festlegt. Es gilt als absehbar, dass Deutschland dieses Ziel verfehlen wird. ${ }^{41}$

\subsubsection{Verordnung über Landnutzung (,LULUCF“)}

Die Verordnung (EU) Nr. 2018/841 über Landnutzungen (sog. LULUCF-Verordnung) ${ }^{42}$ integriert ab 2021 auch Landnutzungsänderungen in den europäischen Klimaschutzrahmen. Sie bezieht sich auf Einbindungen von Emissionen aus Landnutzung, Landnutzungsänderungen und Forstwirtschaft („Land Use, Land Use Change and Forestry“" - „LULUCF“) und legt fest, wie Emissionen und Senken (d.h. $\mathrm{CO}_{2}$-Einbindungen) durch Wälder und Böden verbucht werden. Landnutzung und Forstwirtschaft umfassen die Nutzung von Böden, Bäumen, Pflanzen, Biomasse und Holz. ${ }^{43}$ Der LULUCF-Sektor kann einen besonderen Beitrag zum Klimaschutz leisten, da in diesem nicht nur Treibhausgase ausgestoßen, sondern gleichzeitig auch $\mathrm{CO}_{2}$ aus der Atmosphäre abgebaut werden kann (Senken-Funktion). ${ }^{44}$ Gemäß Art. 4 LULUCF-Verordnung muss jeder Mitgliedstaat für den Zeitraum von 2021 bis 2025 und den Zeitraum von 2026 bis 2030 dafür sorgen, dass die Emissionen nicht den Abbau übersteigen. Auf die EU-Klimaschutzziele wird die Treibhaushausgasbilanz des Landnutzungssektors hingegen nicht unmittelbar angerechnet. ${ }^{45}$ Nach der LULUCFVerordnung hat jeder EU-Mitgliedstaat die tatsächliche $\mathrm{CO}_{2}$-Einbindung von Wäldern und Böden mit in der Verordnung definierten Vergleichsmaßstäben in Beziehung zu setzen (vgl. Art. 5 ff.). Eine Abnahme der $\mathrm{CO}_{2}$-Einbindung gegenüber dem Vergleichsmaßstab führt zu Lastschriften, eine Zunahme zu Gutschriften. ${ }^{46}$ Um die Klimaschutzleistung von Böden zu ermitteln, ist die Treibhausgasbilanz mit dem Zeitraum 2005 bis $2009 \mathrm{zu}$ vergleichen (vgl. Art. 7). Für den bewirtschafteten Wald ist der Berechnung ein Referenzwert zu Grunde zulegen, der von den Mitgliedstaaten an Hand der Kriterien in Anhang IV Abschnitt A festzulegen ist (Art. 8). Bis Ende 2018 hatten die Mitgliedstaaten für den Zeitraum von 2021 bis 2025 einen Anrechnungsplan für die Forstwirtschaft vorzulegen, der auch den Vorschlag für den Referenzwert der Wälder umfasst. ${ }^{47}$ Letztlich müssen die Mitgliedstaaten sicherstellen, dass sie im Saldo mehr Gut- als Lastschriften verbuchen (sog. „No-Debit“-Regel bzw. „Verbot der Minusbilanz“) ${ }^{48}$ Eine entsprechende Verpflichtung existierte teilweise bereits bis 2020 im Rahmen des Kyoto-Protokolls (vgl. Art. 3 Abs. 3 und 4) ${ }^{49}$. Durch die

28) Vgl. Art. 2 Abs. 2 der Richtlinie 2018/410/EU.

29) Vollmer (Fn. 23), 367.

30) Verordnung (EU) Nr. 2018/842 des Europäischen Parlaments und des Rates vom 30. Mai 2018 zur Festlegung verbindlicher nationaler Jahresziele für die Reduzierung der Treibhausgasemissionen im Zeitraum 2021 bis 2030 als Beitrag zu Klimaschutzmaßnahmen zwecks Erfüllung der Verpflichtungen aus dem Übereinkommen von Paris, ABl. EU, 19.6.2018, L 156, S. 26.

31) EU-Kommission, Lastenteilung 2021-2030, https://ec.europa. eu/clima/policies/effort/regulation_de (Zugriff: 16.2.2020).

32) EU-Kommission, Lastenteilung: Emissionsziele der Mitgliedstaaten, https://ec.europa.eu/clima/policies/effort_de (Zugriff: 16.2.2020).

33) Saurer (Fn. 5), 1576.

34) 2. Erwägungsgrund der Klimaschutz-VO, Verheyen/Pabsch, Warum EU-Recht ein deutsches Klimaschutzgesetz unabdingbar macht. Stellungnahme zum Entwurf für ein deutsches Klimaschutzgesetz vom Februar 2019, 9.4.2019, S. 4.

35) Agora Energiewende/Agora Verkehrswende, Die Kosten von unterlassenem Klimaschutz für den Bundeshaushalt, 2018, S. 17.

36) Hierzu auch Agora Energiewende/Agora Verkehrswende (Fn. 35), S. $21 \mathrm{f}$.

37) BT-Drs. 19/14337, S. 17; s. auch die weiteren Flexibilisierungsmöglichkeiten gemäß Art. 5 bis 7 Klimaschutz-VO.

38) ABl. EU, L 165, 18.6.2013, S. 13.

39) Agora Energiewende/Agora Verkehrswende (Fn. 35), S. 25.

40) Entscheidung Nr. 406/2009/EG des Europäischen Parlaments und des Rates vom 23.4.2009 über die Anstrengungen der Mitgliedstaaten zur Reduktion ihrer Treibhausgasemissionen mit Blick auf die Erfüllung der Verpflichtungen der Gemeinschaft zur Reduktion der Treibhausgasemissionen bis 2020, ABl. EU, L 156, S. 1.

41) BT-Drs. 19/14337, S. 17.

42) Verordnung (EU) 2018/841 des Europäischen Parlaments und des Rates vom 18. 5. 2018 über die Einbeziehung der Emissionen und des Abbaus von Treibhausgasen aus Landnutzung, Landnutzungsänderungen und Forstwirtschaft in den Rahmen für die Klima- und Energiepolitik bis 2030, ABl. EU, L 156/1.

43) EU-Kommission, Vorschlag für die Einbeziehung der Landnutzung in den Rahmen für die Klima- und Energiepolitik der EU bis 2030 (Factsheet), 20.7.2016, S. 1.

44) EU-Kommission (Fn. 43).

45) BMU, Klima- und Energiepolitik der Europäischen Union, 5.2.2019, https://www.bmu.de/themen/klima-energie/klimaschutz/eu-klimapolitik/ (Zugriff: 16.2.2020).

46) BMU (Fn. 45).

47) S. hierzu den deutschen Anrechnungsplan: Rock et al., National Forestry Accounting Plan, 17.12.2018.

48) BMU (Fn. 45).

49) Zur Berechnungsmethode siehe Beschluss Nr. 529/2013/EU, ABl. EU, L 165, S. 80. 
LULUCF-Verordnung wird diese Verpflichtung für den Zeitraum 2021-2030 nun erstmals im EU-Recht verankert. ${ }^{50}$ Zudem wird der Anwendungsbereich ausgeweitet: Während bisher nur Wälder umfasst waren, ist die Verordnung auf alle Landnutzungen (ab 2026 zudem auch auf Feuchtgebiete) anwendbar (Art. 2). ${ }^{51}$ Ähnlich wie bei der Klimaschutz-VO sieht die LULUCF-Verordnung Flexibilitätsregelungen für die Mitgliedstaaten vor (Art. 12 und 13). So dürfen Gutschriften in begrenztem Umfang in die Sektoren übertragen werden, die der Klimaschutz-VO unterliegen. Des Weiteren können die Mitgliedstaaten untereinander Gutschriften („Nettoabbau“) kaufen oder verkaufen. Dies schafft für die Mitgliedstaaten einen Anreiz, den $\mathrm{CO}_{2}$-Abbau überobligatorisch zu steigern. ${ }^{52}$

\subsubsection{Europäische Governance-Verordnung}

Die Verordnung (EU) Nr. 2018/1999 (Europäische Governance-Verordnung) ${ }^{53}$ ist ein neues Instrument zur Fortschrittskontrolle in der europäischen Klima- und Energiepolitik. ${ }^{54}$ Sie ist Ende 2018 in Kraft getreten und soll zu einer stärkeren Integration von Energie- und Klimapolitik beitragen. Der Governance-Mechanismus stützt sich auf LangfristStrategien, integrierte nationale Energie- und Klimapläne, Fortschrittsberichte der Mitgliedstaaten sowie Überwachungsmodalitäten der EU-Kommission (Art. 1 Abs. 1 S. 2 Governance-VO). In den integrierten nationalen Energieund Klimaplänen (integrated national energy and climate plans - NECPs, Art. 3 ff.) sollen die Mitgliedstaaten ihre nationale Energie- und Klimapolitik für einen 10-jährigen Zeitraum umfassend darstellen. Die NECPs beziehen sich auf den Zeitraum von 2021 bis 2030 und decken die fünf Dimensionen der EU-Energieunion ${ }^{55} \mathrm{ab}$. Letztere gehen über den reinen Klimaschutz hinaus, sind mit diesem aber eng verbunden. ${ }^{56}$ Für die genannten Bereiche sind die nationalen Ziele sowie nationale Beiträge zu den EU-Zielen sowie die Strategien und Maßnahmen zu beschreiben (vgl. Art. 4 bis 6). Mit Projektionen bestehender Maßnahmen und Folgeabschätzungen geplanter Maßnahmen stellen die Pläne zudem die aktuelle Situation und künftige Entwicklungen im Energieund Klimabereich dar (vgl. Art. 7 und 8). Die Mitgliedstaaten mussten den Entwurf ihres NECP bis zum 31. Dezember 2018 an die EU-Kommission übermitteln (Art. 9). Dieser Anforderung ist das zuständige Bundeswirtschaftsministerium auf der Basis der bis dahin vorliegenden politischen Weichenstellungen nachgekommen (u.a. waren die Ergebnisse der Kohlekommission noch nicht enthalten). ${ }^{57}$ Die EUKommission hat die Entwürfe der Mitgliedstaaten geprüft und Empfehlungen erteilt, denen in den finalen Fassungen der NECP's gebührend Rechnung zu tragen ist (Art. 9 Abs. 3) ${ }^{58}$ Die finale Fassung des NECP musste der EU-Kommission bis Ende 2019 vorgelegt werden (Art. 3 Abs. 1). Die nach Art. 15 Governance-VO geforderte Langfriststrategie für den Klimaschutz hat die Bundesrepublik Deutschland mit dem Klimaschutzplan 2050 bereits beschlossen. ${ }^{59}$

\subsubsection{Vorschlag für ein europäisches Klimaschutzgesetz}

Im Rahmen des sog. Green Dea ${ }^{60}$ hat die EU-Kommission im März 2020 den Vorschlag für ein europäisches Klimaschutzgesetz vorgelegt. ${ }^{61}$ In diesem Gesetz sollen das Ziel der Klimaneutralität bis 2050 verankert und die Richtung für die gesamte zukünftige EU-Klimapolitik festgelegt werden. Der Vorschlag soll den bestehenden politischen Rahmen ergänzen. Die EU-Institutionen und die Mitgliedstaaten werden darin verpflichtet, die notwendigen Maßnahmen zu ergreifen, um das Ziel der Treibhausgasneutralität zu erreichen. Hierbei geht es darum, den Fortschritt zu verfolgen und Maßnahmen entsprechend anzupassen. Dies soll auf Basis bestehender Systeme wie dem Governance-Prozess für die nationalen Energie- und Klimapläne ${ }^{62}$, regelmäßigen Berichten der Europäischen Umweltagentur und den wissenschaftlichen Erkenntnissen über den Klimawandel und seine Auswirkungen geschehen (vgl. Erwägungs- gründe $17 \mathrm{ff}$. des Kommissionsvorschlages). Um das Ziel der Klimaneutralität zu erreichen soll die EU-Kommission bis September 2020 Möglichkeiten zur Reduzierung der Treibhausgasemissionen um 50 bis $\% 55 \%$ bis 2030 gegenüber 1990 prüfen (Art. 2 Abs. 3 des Kommissionsvorschlages). Dies bedeutet eine Verschärfung gegenüber dem bisherigen Ziel der Senkung der THG-Emissionen (mindestens 40\% gegenüber dem Jahr 1990, vgl. Art. 2 Nr. 11 GovernanceVO). Bis zum 30. Juni 2021 soll die EU-Kommission zudem alle relevanten Rechtsvorschriften überprüfen und ggf. deren Überarbeitung vorschlagen, um die anvisierten Emissionsreduktionsziele $\mathrm{zu}$ erreichen (Art. 2 Abs. 4). Weiterhin sieht der Kommissionsvorschlag eine Ermächtigung an die EU-Kommission vor, durch delegierte Rechtsakte einen EU-weiten Pfad für die Verringerung der Treibhausgasemissionen für den Zeitraum 2030-2050 festzulegen (Art. 3 und Art. 9). Da es sich hierbei um sehr weitreichende Entscheidungen handelt ist fraglich, ob die Mitgliedstaaten dieser Regelung zustimmen. Bis September 2023 und danach alle fünf Jahre soll die EU-Kommission die Entwicklung bewerten und bei Bedarf Anpassungen vorschlagen (Art. 5). Die Kommission soll auch befugt werden, Empfehlungen an die Mitgliedstaaten auszusprechen, deren Maßnahmen nicht mit dem Ziel der Klimaneutralität in Einklang stehen. Die Mitgliedstaaten werden verpflichtet, diesen Empfehlungen gebührend Rechnung zu tragen oder zu begründen, falls sie dies nicht tun (Art. 6). Neben diesen Maßnahmen des Klimaschutzes ist auch eine Intensivierung der Anpassungsbemühungen an den Klimawandel vorgesehen. Demnach sollen die Mitgliedstaaten Anpassungsstrategien und -pläne erstellen, die den Rahmen für ein umfassendes Risikomanagement enthalten, und diese durchführen (Art. 4). Maßnahmen zur Öffentlichkeitsbeteiligung sind in Art. 8 des Kommissionsvorschlages geregelt. Der weitere Fortgang des Gesetzgebungsverfahrens bleibt abzuwarten.

\section{Klimaschutzgesetz des Bundes}

Im Folgenden werden der Entstehungsprozess und die inhaltlichen Vorgaben des Bundes-Klimaschutzgesetzes (KSG)

50) EU-Kommission, Vorschlag für die Einbeziehung der Landnutzung in den Rahmen für die Klima- und Energiepolitik der EU bis 2030, 20.7.2016, S. 2.

51) EU-Kommission, Verordnung über Landnutzung und Forstwirtschaft für 2021-2030, https://ec.europa.eu/clima/policies/ forests/lulucf_de (Zugriff: 16.2.2020).

52) EU-Kommission (Fn. 51)

53) Verordnung (EU) 2018/1999 des Europäischen Parlaments und des Rates vom 11. Dezember 2018 über das Governance-System für die Energieunion und für den Klimaschutz, ABl. L 328 vom 21. 12.2018, S. 1

54) Ausführlich hierzu acatech/Leopoldina/Akademienunion (Hrsg.), Governance für die Europäische Energieunion, 2018.

55) Sicherheit der Energieversorgung, Energiebinnenmarkt, Energieeffizienz, Dekarbonisierung sowie Forschung, Innovation und Wettbewerbsfähigkeit (vgl. Art. 4)

56) Verheyen/Pabsch (Fn. 34), S. 5.

57) BMWi, Nationaler Energie- und Klimaplan (NECP), Entwurf des integrierten nationalen Energie- und Klimaplans, 4. 1.2019.

58) Z.B. Empfehlung der Kommission vom 18.6.2019 zum Entwurf des integrierten nationalen Energie-und Klimaplans Deutschlands für den Zeitraum 2021-2030, COM(2019) 4405 final; s. auch die Stellungnahmen von Schlacke, https://energiesysteme-zukunft.de/themen/debatte/klimaschutzgesetz/ (Zugriff: 16.2.2020) sowie Schlacke/Knodt, ZUR 2019, 404, 408.

59) S. hierzu ausführlich unten 3.2.6.

60) Mitteilung der Kommission: Der europäische Grüne Deal, COM(2019) 640 final vom 11. Dezember 2019.

61) Vorschlag für eine Verordnung des europäischen Parlaments und des Rates zur Schaffung des Rahmens für die Verwirklichung der Klimaneutralität und zur Änderung der Verordnung (EU) 2018/1999 (Europäisches Klimagesetz), 4.3.2020, COM(2020) 80 final.

62) Siehe hierzu oben 2.2.4. 
erläutert. Hierbei zeigt sich, dass die Vorschriften des KSG in vielerlei Hinsicht auf den europäischen und internationalen Vorgaben aufbauen bzw. diese umsetzen.

\subsection{Entstehungsprozess}

Ausgangspunkt der Entstehung des KSG ist das Energiekonzept der Bundesregierung vom 28.9.2010, welches bereits konkrete THG-Minderungsziele enthält. ${ }^{63}$ Bemühungen um ein Bundesklimaschutzgesetz nach dem Vorbild des UK Climate Change Act $2008^{64}$ waren zu dieser Zeit noch nicht erfolgreich. ${ }^{65}$ Allerdings wurden daraufhin in einigen Bundesländern entsprechende allgemeine Klima(schutz)gesetze verabschiedet. Vorreiter war Nordrhein-Westfalen (2013) ${ }^{66}$, es folgten Baden-Württemberg (2013) ${ }^{67}$, Rheinland-Pfalz $(2014)^{68}$, Bremen $(2015)^{69}$, Berlin $(2016)^{70}$, Schleswig-Holstein $(2017)^{71}$ und Thüringen $(2018)^{72}$. In Niedersachsen befindet sich ein entsprechender Gesetzentwurf im Gesetzgebungsverfahren. ${ }^{73}$ Hamburg hatte bereits 1997 ein Klimaschutzgesetz mit detaillierten Maßnahmen vorge$\operatorname{legt}^{74}$, allerdings ohne konkrete Klimaschutzziele. Auf Bundesebene sah der Koalitionsvertrag zwischen CDU, CSU und SPD für die 18. Legislaturperiode die Verabschiedung eines Klimaschutzplans vor. ${ }^{75}$ Der sog. „Klimaschutzplan 2050“ wurde am 14. November 2016 durch das Bundeskabinett beschlossen. ${ }^{76}$ Der Koalitionsvertrag für die laufende 19. Legislaturperiode stellte für das Jahr 2019 die Verabschiedung eines Klimaschutzgesetzes in Aussicht. ${ }^{77}$

Daraufhin hat das BMU im Februar 2019 den Entwurf eines Klimaschutzgesetzes (Referentenentwurf) zur Information und frühzeitigen Stellungnahme an das Bundeskanzleramt übersandt. ${ }^{78}$ Wenig später einigte sich der Koalitionsausschuss auf die Einrichtung eines Klimakabinetts, in dem verschiedene Fachminister konkrete Maßnahmen für ihren jeweiligen Bereich zusammentragen sollen. ${ }^{79}$ Nach der Europawahl vom 27. Mai 2019, die auch als Klimawahl interpretiert wurde, brachte das BMU den o.g. Gesetzentwurf in die Ressortabstimmung ein. ${ }^{80} \mathrm{Am}$ 20. September 2019 wurden durch das Klimakabinett im Rahmen des sog. „Klimapakets“ maßgebliche Eckpunkte für ein Klimaschutzprogramm ${ }^{81}$ vorgelegt. $^{82}$ Darin war auch die politische Einigung auf zentrale Elemente eines Klimaschutzgesetzes enthalten. ${ }^{83}$ Zeitgleich mit dem sog. Klimaschutzprogramm 2030 ${ }^{84}$, hat das Bundeskabinett am 9. Oktober 2019 den (gegenüber der Version von Februar überarbeiteten) Entwurf für ein Klimaschutzgesetz beschlossen. ${ }^{85}$ Nach letzten Änderungen durch den Umweltausschuss ${ }^{86}$ nahm der Bundestag das (durch den Bundesrat nicht zustimmungspflichtige) Gesetz am 15 . November an. ${ }^{87}$ Das KSG ist am 18. Dezember 2019 in Kraft getreten. ${ }^{88}$

Die nunmehr bestehende Motivation für ein solches Klimaschutzgesetz liegt ausweislich der Gesetzesbegründung darin, dass Deutschland sein nationales Klimaschutzziel voraussichtlich schon für 2020 verfehlen werde. ${ }^{89}$ Dies mache vor dem Hintergrund der bestehenden internationalen und europäischen Verpflichtungen verstärkte Klimaschutzanstrengungen erforderlich. Gleichzeitig gehe es auch darum, die aus der Zielverfehlung resultierenden drohenden erheblichen Belastungen des Bundeshaushalts abzuwenden. Diese ergeben sich aus Zahlungspflichten für die Verfehlung der unionsrechtlich verbindlichen Ziele in den Sektoren Verkehr, Gebäude, Industrie und Landwirtschaft. ${ }^{90}$ Denn bei einer Überschreitung dieser Emissionsziele muss Deutschland das Defizit durch Erwerb von Emissionszuweisungen aus andern Mitgliedstaaten ausgleichen. ${ }^{91} \mathrm{Ab}$ 2021 gelten für die genannten Sektoren nochmals erheblich verschärfte Vorgaben (vgl. Verpflichtungen der Klimaschutz-VO). Für die verstärkten Klimaschutzanstrengungen sieht die Bundesregierung neben Einzelmaßnahmen in den einzelnen Sektoren einen übergreifenden Rahmen für erforderlich an. Eine gesetzliche Regelung der erforderli- chen Klimaschutzziele mit jährlich absinkenden, zulässigen Jahresemissionsmengen gewährleiste eine bessere Vorhersehbarkeit und Planbarkeit für die betroffenen Akteure. ${ }^{92}$

\subsection{Inhaltliche Vorgaben des KSG}

Das Bundesklimaschutzgesetz (KSG) enthält 15 Paragraphen und ist in fünf Abschnitte unterteilt.

63) BMWi (Hrsg.), Energiekonzept für eine umweltschonende, zuverlässige und bezahlbare Energieversorgung, 28.9.2010, S. 5 .

64) Climate Change Act 2008, http://www.legislation.gov.uk/ukpga/2008/27/contents (Zugriff: 16.2.2020); näher hierzu Rodi/ Sina, Das Klimaschutzrecht des Bundes - Analyse und Vorschläge zu seiner Weiterentwicklung, 2010 (UBA, Climate Change 17/11 sowie Saurer, NuR 2018, 581, 583.

65) Sina, EurUP 2018, 314, 323.

66) Gesetz zur Förderung des Klimaschutzes in NRW v. 29.1.2013, GV. NRW Nr. 4, S. 29.

67) Gesetz zur Förderung des Klimaschutzes in Bad.-Württ. v. 23.7.2013, GBl. BW Nr. 11, S. 229.

68) Landesgesetz zur Förderung des Klimaschutzes (Landesklimaschutzgesetz - LKSG) v. 19. 8.2014, GVBl. S. 188.

69) Bremisches Klimaschutz- und Energiegesetz (BremKEG) v. 24.3.2015, GBl. S. 124.

70) Energiewendegesetz Berlin (EWG Bln) v. 5.4.2016, GVBl., S. 122.

71) Energiewende- und Klimaschutzgesetz Schleswig-Holstein (EWKG) v. 7.3.2017, GVBl. S. 124.

72) Thüringer Gesetz zum Klimaschutz und zur Anpassung an die Folgen des Klimawandels (Thüringer Klimagesetz - ThürKli$\mathrm{maG})$ v. 18.12.2018, GVBl. S. 816.

73) Niedersächsisches Gesetz zur Förderung des Klimaschutzes und zur Anpassung an die Folgen des Klimawandels (Niedersächsisches Klimagesetz - Nds. KlimaG), LT-Drs. 18/4499.

74) Hamburgisches Gesetz zum Schutz des Klimas durch Energieeinsparung (Hamburgisches Klimaschutzgesetz - HmbKliSchG) v. 25. 6. 1997, GVBl. S. 261.

75) Deutschlands Zukunft gestalten. Koalitionsvertrag zwischen CDU, CSU und SPD, Berlin, 14. Dezember 2013, S. 37.

76) BMU, Klimaschutzplan 2050 (Fn. 16).

77) Ein neuer Aufbruch für Europa. Eine neue Dynamik für Deutschland. Ein neuer Zusammenhalt für unser Land, Koalitionsvertrag zwischen CDU, CSU und SPD, Berlin, den 12.3.2018.

78) Der Gesetzentwurf ist abrufbar unter: https://www.klimareporter.de/images/dokumente/2019/02/ksg.pdf (Zugriff: 1.9.2019).

79) Bundesregierung, Einrichtung eines Klimakabinetts, 20.3.2019, https://www.bundesregierung.de/breg-de/aktuelles/bundesregierung-packt-klimaschutz-an-1592188 (Zugriff: 16.2.2020).

80) BMU, Meldung vom 27.5.2019, https://www.bmu.de/meldung/svenja-schulze-treibt-klimaschutzgesetz-voran/ (Zugriff: 16.2.2020).

81) Eckpunkte für ein Klimaschutzprogramm (Fassung nach Klimakabinett).

82) BMU, Pressemitteilung Nr.160/19, https://www.bmu.de/pressemitteilung/schulze-beschluesse-des-klimakabinetts-markierenneuanfang-fuer-deutsche-klimapolitik/ (Zugriff: 16.2.2020).

83) Eckpunkte für ein Klimaschutzprogramm (Fn. 81), S. $20 \mathrm{f}$.

84) Klimaschutzprogramm 2030 der Bundesregierung zur Umsetzung des Klimaschutzplans 2050, Stand: 8.10.2019.

85) BMU, Pressemitteilung Nr. 173/19; https://www.bmu.de/ pressemitteilung/schulze-klimaschutz-wird-gesetz/ (Zugriff: 16.2.2020); BT-Drs.19/14948 (Gesetzentwurf der Bundesregierung), textidentisch mit BT-Drs. 19/14337 (Gesetzentwurf der Fraktionen der CDU/CSU und SPD).

86) BT-Drs. 19/15128, S. 5.

87) Deutscher Bundestag, Bundestag nimmt das Klimapaket der Koalition an, https://www.bundestag.de/dokumente/textarchiv/2019/ kw46-de-klimaschutzgesetz-freitag-667244 (Zugriff: 16.2.2020).

88) Gesetz zur Einführung eines Bundesklimaschutzgesetzes und zur Änderung weitere Vorschriften vom 12.12.2019, BGBl. I, 2513.

89) BT-Drs. 19/14337, S. 1 f. S. aber zu den aktuellen Entwicklungen durch die Corona-Pandemie: Hein/Peter/Graichen, Auswirkungen der Corona-Krise auf die Klimabilanz Deutschlands, März 2020.

90) Siehe oben Kap. 2.2.2.

91) BT-Drs. 19/14337, S. 1.

92) BT-Drs. 19/14337, S. 2. 


\subsubsection{Gesetzeszweck, Begriffsdefinitionen}

Abschnitt 1 des KSG („Allgemeine Vorschriften“) regelt den Gesetzeszweck $(\$ 1)$ und Begriffsbestimmungen $(\$ 2)$. Gemäß $\ 1$ Abs. 1 S. 1 KSG ist die Intention des Gesetzes darauf ausgerichtet, zum Schutz vor den Auswirkungen des weltweiten Klimawandels die Erfüllung der nationalen Klimaschutzziele sowie die Einhaltung der europäischen Zielvorgaben zu gewährleisten. Als Grundlagen werden insoweit die Verpflichtung nach dem Paris Agreement zur Begrenzung des Temperaturanstiegs auf 2 bzw. 1,5 Grad Celsius sowie das Bekenntnis Deutschlands auf dem UN Klimagipfel von New York vom 23. September 2019, Treibhausgasneutralität bis 2050 als langfristiges Ziel zu verfolgen, genannt. (Netto-) Treibhausgasneutralität wird in $\$ 2 \mathrm{Nr} .9$ definiert als das Gleichgewicht zwischen den anthropogenen Emissionen von Treibhausgasen als Quellen und dem Abbau solcher Gase durch Senken. Der Begriff Treibhausgase ist in $\$ 2 \mathrm{Nr}$. $1 \mathrm{KSG}$ näher bestimmt und umfasst u.a. die Stoffe Kohlendioxid $\left(\mathrm{CO}_{2}\right)$, Methan $\left(\mathrm{CH}_{4}\right)$ und Di-stickstoffoxid $\left(\mathrm{N}_{2} \mathrm{O}\right)$.

\subsubsection{Klimaschutzziele}

Herzstück des KSG ist dessen Abschnitt 2 („Klimaschutzziele und Jahresemissionsmengen“). Zentrale Norm ist $\$ 3$, der die nationalen Klimaschutzziele für das Jahr 2030 festlegt. Demnach werden die THG-Emissionen in Deutschland im Vergleich zum Jahr 1990 schrittweise gemindert, dabei um mindestens 55 Prozent bis zum Zieljahr 2030. Hiermit wird das Minderungsziel übernommen, welches bereits im Klimaschutzkonzept 2010 und dem Klimaschutzplan 2016 festgelegt ist. Es ist nahezu deckungsgleich mit dem auf Deutschland entfallenden Anteil an der Erreichung des europäischen Klimaschutzziels für $2030 .{ }^{93} \mathrm{Kli}-$ maschutzziele für das Jahr 2020 sowie die Jahre 2040 und 2050 sind im Gegensatz zu der Fassung des Referentenentwurfs nicht mehr im Gesetz enthalten. Für die Zeiträume ab dem Jahr 2031 sieht $\$ 4$ Abs. 1 S. 5 KSG daher die Fortschreibung der jährlichen Minderungsziele durch eine Rechtsverordnung der Bundesregierung mit Zustimmung des Bundestages auf der Grundlage von $\$ 4$ Abs. 6 KSG vor. Das Ziel der Erreichung der Netto-Treibhausgasneutralität bis zum Jahr 2050 wird allerdings in $\$ 1 \mathrm{KSG}$ als Gesetzeszweck genannt.

\subsubsection{Jahresemissionsmengen}

Zur Erreichung der o.g. Klimaschutzziele werden in $\$ 4$ KSG jährliche Minderungsziele in Form von zulässigen Jahresemissionsmengen für in der Anlage 1 aufgeführte Sektoren (Energiewirtschaft, Industrie, Verkehr, Gebäude, Landwirtschaft, Abfallwirtschaft und Sonstiges) festgelegt. Im Einklang mit den internationalen bzw. europäischen Vorgaben nicht erfasst sind Emissionen aus dem LULUCFSektor sowie Emissionen des Luft- und Seeverkehrs, die anderweitigen Anforderungen unterliegen. ${ }^{94}$ Für die einzelnen Sektoren enthält Anlage 2 für den Zeitraum bis zum Jahr 2030 (in der Regel jährlich) ${ }^{95}$ abssinkende Treibhausgasemissionsmengen in Tonnen $\mathrm{CO}_{2}$ - Äquivalent ${ }^{96}(\mathbb{S} 4$ Abs. 1 S. 3 KSG). Diese ergeben sich aus den Sektorzielen des Klimaschutzplans und entsprechen für die von der europäischen Klimaschutz-VO erfassten Sektoren den unionsrechtlichen Anforderungen. ${ }^{97}$ Anders als bei Punktzielen für bestimmte Zieljahre kommt es insoweit nicht allein darauf an, das Emissionsniveau in einem Zieljahr zu erreichen; maßgeblich ist vielmehr das gesamte Emissionsvolumen im zehnjährigen Zeitraum bis zum Jahr 2030. ${ }^{98}$ Für die Einhaltung der Jahresemissionsmengen ist gemäß §4 Abs. $4 \mathrm{KSG}$ das für einen Sektor überwiegend zuständige Bundesministerium verantwortlich und hat die Aufgabe, die erforderlichen nationalen Maßnahmen zu veranlassen. Die Zuordnung der zulässigen THG-Jahresemissionsmengen $\mathrm{zu}$ den verantwortlichen Sektoren bzw. Ministerien ist auch im internationalen Vergleich Neuland ${ }^{99}$ und fördert die Einhaltung des jährlich reduzierten Budgets. ${ }^{100}$ Die Bundesregierung wird in $\$ 4$ Abs. 5 S. 1 KSG ermächtigt, durch Rechtsverordnung die Jahresemissionsmengen der Sektoren mit Wirkung zum Beginn des jeweils nächsten Kalenderjahres zu ändern, was der Bundesregierung mehr Flexibilität bei der Zielerreichung einräumt. ${ }^{101}$ Die Änderungen müssen im Einklang mit der Erreichung der nationalen Klimaschutzziele und mit den europarechtlichen Anforderungen stehen ( $\$ 4$ Abs. 5 S. 2 KSG).

3.2.4 Erstellung der Emissionsdaten durch das Umweltbundesamt

\5 KSG regelt die Berichterstattung. Das Umweltbundesamt (UBA) veröffentlicht und berichtet dem Expertenrat für Klimafragen (vgl. \$10 KSG) jährlich im März jedes Jahres die Emissionsdaten des vergangenen Jahres für die o.g. Sektoren ( $\$ 5$ Abs. $1 \mathrm{KSG})$. Die Vorschrift knüpft an die bisherige jährliche Berichterstattung des UBA über die vorläufigen nationalen Treibhausgasinventare nach der UN-Klimarahmenkonvention und der Europäischen Klimaberichterstattungsverordnung ${ }^{102}$ an. $\mathrm{Ab}$ dem Berichtsjahr 2021 werden weitere Informationen dargestellt, u.a. Über- oder Unterschreitungen der Jahresemissionsmengen nach Anlage 2 sowie im Hinblick auf den LULUCF-Sektor auch Quellen und Senken von Treibhausgasen (vgl. $\int 5$ Abs. 2 Nrn. 1 bis 4 KSG). Das UBA darf die zur Aufgabenerfüllung erforderlichen Daten erheben und die Bundesregierung wird ermächtigt, das Nähere in einer Rechtsverordnung zu regeln ( $\$ 5$ Abs. 3 und $4 \mathrm{KSG}$ ). Verstöße gegen die Verordnung oder gegen vollziehbare Anordnungen auf Grund der Verordnung sind bußgeldbewehrt ( $\$ 6 \mathrm{KSG}$ ). Hiermit wird dem Erfordernis der Vollständigkeit, Richtigkeit und rechtszeitigen Verfügbarkeit der Emissionsdaten Rechnung getragen. ${ }^{103}$

3.2.5 Sofortprogramm bei Überschreitung der Emissionsmenge

$\$ 8$ KSG bestimmt, dass die Bundesregierung ein Sofortprogramm auflegen und umsetzen muss, sofern Emissionsdaten nach $\$ 5$ eine Überschreitung der zulässigen Jahresemissionsmenge für einen Sektor in einem Berichtsjahr ausweisen (Abs. 1). Das Programm soll die Einhaltung der Jahresemissionsmengen des Sektors für die folgenden Jahre sicherstellen und der Bundesregierung innerhalb von drei Monaten nach der Bewertung der Emissionsdaten durch den Expertenrat vorgelegt werden. Durch das Monitoring der Zielerfüllung und die erforderliche Auflegung eines Sofortprogramms wird gewährleistet, dass Zielverfehlungen politisch nicht unbemerkt bleiben und zeitnah nachgesteuert werden kann. ${ }^{104}$ Die Verantwortlichkeit für die Erarbeitung des Programms liegt bei dem für den Sektor zuständigen Bun-

93) BT-Drs. 19/14337, S. 27.

94) Näher hierzu BT-Drs. 19/14337, S. 27 sowie oben 2.

95) Im Sektor Energiewirtschaft sollen die Emissionen im Einklang mit dem Abschlussbericht der Kommission ,Wachstum, Strukturwandel und Beschäftigung" möglichst stetig sinken, wobei für bestimmte Jahre (2020, 2022 und 2030) Jahresemissionsmengen festgelegt werden; BT-Drs. 19/14337, S. 28 und 42.

96) Zur Begriffsdefinition „Treibhausgasemissionen“ s. $\$ 2$ Nr. 2 KSG.

97) BT-Drs. 19/14337, S. 28

98) BT-Drs. 19/14337, S. 28

99) Scharlau et al., NVwZ 2020, 1, 3.

100) Verheyen/Pabsch (Fn. 34), S. 8.

101) Vgl. Verheyen/Pabsch (Fn. 34), S. 16.

102) Durchführungsverordnung (EU) Nr. 749/2014 der Kommission vom 30.6.2014, AB1. EU, L 203, S. 23. Aufgrund von Art. 26 Abs. 7 Governance-Verordnung kann die EU-Kommission eine Nachfolgeregelung erlassen.

103) BT-Drs. 19/14337, S. 31.

104) Verheyen/Pabsch (Fn. 34), S. 10. 
desministerium. Nach Vorlage des Sofortprogramms berät die Bundesregierung über die zu ergreifenden Maßnahmen. Hierbei kann es sich um Maßnahmen im betroffenen Sektor, in anderen Sektoren oder um sektorübergreifende Maßnahmen handeln. Die Maßnahmen sind schnellstmöglich zu beschließen ( 88 Abs. 2 S. 1 KSG). Im Rahmen der Maßnahmenauswahl kann die Bundesregierung die Spielräume der Europäischen Klimaschutz-VO $\mathrm{V}^{105}$ berücksichtigen (insb. die Zulässigkeit der Verrechnung zwischen den Sektoren und zwischen verbindlichen Jahresbudgets ${ }^{106}$ und gem. $\ 4$ Abs. 5 KSG die Jahresemissionsmengen der Sektoren im Verordnungsweg ändern ( $\$ 8$ Abs. 2 S. 2 KSG). Die Vorschrift des \8 KSG ähnelt Art. 8 Abs. 1 der Klimaschutz-VO, wonach die Mitgliedstaaten der EU-Kommission einen Plan für Abhilfemaßnahmen vorlegen müssen, falls sie ihr jährliches nationales Emissionsminderungsziel verfehlen. Im Gegensatz zur Verpflichtung nach Art. 8 Abs. 1 Klimaschutz-VO greift das Sofortprogramm bereits im Rahmen der Zielverfehlung eines einzelnen Sektors. ${ }^{107}$ Allerdings kann auch bereits die Verfehlung eines Sektorziels dazu führen, dass Deutschland sein Jahresbudget für THG-Emissionen überschreitet. ${ }^{108}$ Insofern wird durch $\$ 8 \mathrm{KSG}$ ein zeitlicher Gleichlauf mit Art. 8 Abs. 1 Klimaschutz-VO sichergestellt. ${ }^{109}$

\subsubsection{Klimaschutzplan}

Der dritte Abschnitt des KSG widmet sich der „Klimaschutzplanung" und enthält Vorschriften zum Klimaschutzprogramm $(\$ 9)$ und zur Berichterstattung $(\$ 10)$. Eine eigene Vorschrift zum Instrument des Klimaschutzplans ist im Gegensatz zum Referentenentwurf des KSG vom Februar $2019^{110}$ nicht mehr enthalten. Der Klimaschutzplan wird lediglich im Rahmen der Begriffsbestimmungen des $\$ 2$ Nr. 7 KSG und in $\$ 12$ Abs. 2 KSG erwähnt und i.S.v. Art. 15 Abs. 1 Governance-VO als ,die deutsche Langfriststrategie nach dem Übereinkommen von Paris und nach Artikel 15 der Europäischen Governance-Verordnung“ definiert. Auf Grund der unmittelbaren Geltung der Governance-Verordnung (vgl. Art. 288 AEUV) ist eine nationale Regelung auch nicht angezeigt bzw. entbehrlich. ${ }^{111}$ Klimaschutzpläne sind gem. Art. 15 Abs. 1 Governance-VO bis zum 1. Januar 2020 und anschließend bis zum 1. Januar 2029 und danach alle zehn Jahre von den Mitgliedstaaten mit einer Perspektive von mindestens dreißig Jahren zu erstellen und erforderlichenfalls alle fünf Jahre zu aktualisieren. Die notwendigen Inhalte ergeben sich direkt aus Art. 15 Abs. 4 i. V.m. Anhang IV Governance-VO. Die integrierten nationalen Energie- und Klimapläne i.S. von Art. 3 Governance-Verordnung (NECPs) ${ }^{112}$ sollen auf die Klimaschutzpläne abgestimmt werden (Art. 15 Abs. 6 Governance-VO). In Deutschland liegt mit dem „Klimaschutzplan 2050“ eine Langfriststrategie i.S. von Art. 15 Abs. 1 Governance-VO bereits vor. ${ }^{113}$ Darin wird die weitgehende THG-Neutralität Deutschlands bis zum Jahr 2050 als Langfristziel festgelegt. ${ }^{114}$ Mittelfristiges Ziel ist die Minderung der THGEmissionen bis 2030 um mindestens 55 Prozent gegenüber dem Niveau von 1990. ${ }^{115}$ Die jeweiligen Reduktionsziele bis zum Jahr 2030 werden sektorspezifisch verteilt (Energiewirtschaft, Industrie, Gebäude, Verkehr, Landwirtschaft sowie Landnutzung und Forstwirtschaft), wobei den größten Anteil der Gebäudebereich und die Energiewirtschaft erbringen sollen. ${ }^{116}$ Daran anknüpfend beschreibt der Klimaschutzplan die notwendigen Entwicklungspfade in den einzelnen Sektoren und führt erste Maßnahmen zur Umsetzung auf. Der Klimaschutzplan ist zwar durch das Bundeskabinett beschlossen, aber nicht als Rechtsverordnung erlassen worden. Demgemäß bindet er zwar die Bundesministerien, nicht aber die Bürger, Unternehmen und Gerichte. ${ }^{17}$

\subsubsection{Klimaschutzprogramm}

Im Gegensatz zum Klimaschutzplan ist das Instrument des Klimaschutzprogramms eigenständig im KSG geregelt (vgl. \9). Die Erstellung von Klimaschutzprogrammen ist weder völker- noch europarechtlich vorgesehen. Insbesondere sieht die Klimaschutz-VO kein Maßnahmenprogramm o.̈. vor, sondern fordert lediglich für den Fall der Zielverfehlung ,einen Plan für Abhilfemaßnahmen" (s. hierzu bereits 2.2.2). ${ }^{118}$ Jedoch ist im Klimaschutzplan 2050 vorgesehen, diesen mit „Maßnahmenprogrammen“ zu unterlegen. ${ }^{119}$ In diesem Sinne verpflichtet $\$ 9$ KSG die Bundesregierung, mindestens nach jeder Fortschreibung des Klimaschutzplans ein Klimaschutzprogramm zu beschließen. Aus diesem geht hervor, welche Maßnahmen zur Erreichung der Klimaschutzziele sowie zur Einhaltung der Jahresemissionsmengen in den einzelnen Sektoren $\mathrm{zu}$ ergreifen sind ( $\$ 9$ Abs. 1 S. 2 und $3 \mathrm{KSG}$ ). Zudem hat die Bundesregierung Maßnahmen zum Erhalt und Ausbau der Senkenfunktion des LULUCF-Sektors festzulegen (\$9 Abs. 1 S. 4 KSG). Klimaschutzprogramme, die im Zuge der Fortschreibung des Klimaschutzplans aufgestellt werden, dienen der Umsetzung der mit der Fortschreibung beschlossenen Änderungen und Ergänzungen. ${ }^{120}$ In diesem Rahmen schlagen die für die Sektoren zuständigen Bundesministerien innerhalb von sechs Monaten nach Fortschreibung des Klimaschutzplans Maßnahmen vor, die geeignet sind, die in den jeweiligen Sektoren erforderlichen zusätzlichen THG-Minderungen zu erzielen ( $\$ 9$ Abs. 2 S. 2 KSG). Die Bundesregierung kann aber auch unabhängig von der Fortschreibung der Klimaschutzpläne (weitere) Klimaschutzprogramme beschließen. ${ }^{121}$ Dies kann z.B. erforderlich werden, wenn eine Zielverfehlung in einem Sektor besteht bzw. bereits absehbar ist. Sofern in einem solchen Fall bereits ein Sofortprogramm nach $₫ 8$ KSG beschlossen worden ist, wird eine Aktualisierung des bestehenden Klimaschutzprogramms um Maßnahmen nach $₫ 8$ Abs. 2 KSG vorgenommen ( $\$ 1$ Abs. 1 S. 1 Hs. 2 KSG). Im Gegensatz zum Sofortprogramm, welches ad hoc zur Gegensteuerung bei Zielverfehlung dient, handelt es sich beim Klimaschutzprogramm um das (weiter vorausschauende) Regelinstrument zur Erreichung der Klimaziele. ${ }^{122}$ Grundlage eines Klimaschutzprogramms ist der alle zwei Jahre zu erstellende Klimaschutz-Projektionsbericht nach $\int 10$ Abs. 2 KSG, der eine Schätzung der voraussichtlichen THG-Emissionen der nächsten 20 Jahre enthält. ${ }^{123}$ Dies verdeutlicht den planerisch-strategischen Charakter des Klimaschutzprogramms. ${ }^{124}$ Für jedes Klimaschutzprogramm führt die Bundesregierung ein öffentliches Konsultationsverfahren durch (vgl. \$9 Abs. 2 und 3 KSG). Das erste Klimaschutzprogramm hat die Bundesregierung am 9. Oktober 2019 beschlossen. ${ }^{125}$ Dieses sog. „Klimaschutzprogramm 2030“ dient der Umsetzung des Klimaschutzplans 2050 und soll die Erreichung der Klimaziele für das Jahr 2030 sicherstellen. ${ }^{126}$ Es umfasst neben sektorenübergreifenden Klimaschutzansätzen
105) Näher hierzu oben Kap. 2.2.2.
106) BT-Drs. 19/14337, S. 32.
107) BT-Drs. 19/14337, S. 31
108) Verheyen/Pabsch (Fn. 34), S. 9.
109) BT-Drs. 19/14337, S. 32.
110) Vgl. $\$ 9$ des Referentenentwurfs.
111) Verheyen/Pabsch (Fn. 34), S. $7 f$
112) Siehe oben Kap. 2.2.4.
113) Siehe oben Kap. 2.2.4
114) BMU, Klimaschutzplan 2050 (Fn. 16), S. 28.
115) BMU, Klimaschutzplan 2050 (Fn. 16), S. 32f.

116) Gebäudebereich: $67-66 \%$, Energiewirtschaft: $62-61 \%$, Verkehr: 42-40\%; Industrie: 51-49\%; Landwirtschaft: 34-31\%; Sonstige: 87\%; BMU, Klimaschutzplan 2050 (Fn. 16), S. 33.

117) Saurer (Fn. 5), 1578

118) Verheyen/Pabsch (Fn. 34), S. 8.

119) BMU, Klimaschutzplan 2050 (Fn. 16), S. 9.

120) BT-Drs. $19 / 14337$, S. 33

121) BT-Drs. 19/14337, S. 33.

122) BT-Drs. 19/14337, S. 32, 33; Scharlau et al. (Fn. 99), 3.

123) BT-Drs. 19/14337, S. 34; s. hierzu auch unten Kap. 3.2.8

124) Scharlau et al. (Fn. 99), 3.

125) Klimaschutzprogramm 2030 (Fn. 84).

126) BT-Drs. 19/14337, S. 33. 
in Form einer $\mathrm{CO}_{2}$-Bepreisung und Maßnahmen zur Entlastung vor Bürgern und Wirtschaft verschiedene sektorale Maßnahmen in den Sektoren laut Klimaschutzplan 2050 (Energiewirtschaft, Gebäude, Verkehr, Industrie, Landwirtschaft) und Abfallwirtschaft. Hinzu kommen Maßnahmen im LULUCF-Sektor sowie übergreifende Maßnahmen aus den Bereichen klimaneutrale Bundesverwaltung, sustainable finance, Forschung und Innovation sowie Klimaschutz und Gesellschaft. Die Umsetzung der Maßnahmen erfordert die Änderung verschiedener Fachgesetze sowie die Auflegung von Förderprogrammen.

\subsubsection{Berichterstattung}

\$10 KSG normiert die Klimaschutz-Berichterstattung und greift dabei zwei seit längerem bewährte Berichtsformate auf nationaler und europäischer Ebene auf. Abs. 1 regelt die jährliche Erstellung der Klimaschutzberichte durch die Bundesregierung, welche bereits bisher auf Grundlage des „Aktionsprogramms Klimaschutz 2020“ aufzustellen waren. ${ }^{127}$ Die Klimaschutzberichte enthalten Angaben zur Entwicklung der THG-Emissionen in den verschiedenen Sektoren, den Stand der Umsetzung der Klimaschutzprogramme nach $\$ 9$ und der Sofortprogramme nach $\$ 8$ sowie eine Prognose der zu erwartenden THG-Minderungswirkungen ( $\$ 10$ Abs. 1 S. 1 KSG). Die Berichte sind somit ein zentrales MonitoringInstrument des Klimaschutzes in Deutschland. ${ }^{128}$ Des Weiteren hat die Bundesregierung ab dem Jahr 2021 alle zwei Jahre einen Klimaschutz-Projektionsbericht nach den Vorgaben des Art. 18 Governance-VO zu erstellen (bisher war ein inhaltsgleicher Bericht auf Grundlage der Verordnung (EU) Nr. 525/2013 ${ }^{129}$ vorzulegen). Der Bericht enthält die Projektionen von THG-Emissionen (einschließl. der Quellen und Senken des LULUCF-Sektors) und die nationalen Politiken und Maßnahmen zu deren Minderung (Art. 10 Abs. 2 KSG).

\subsubsection{Expertenrat für Klimafragen}

Der vierte Abschnitt des KSG regelt die Einrichtung eines unabhängigen Expertenrats für Klimafragen (\$11) und dessen Aufgaben ( $\$ 12)$. Der Expertenrat besteht aus fünf sachverständigen Personen verschiedener Disziplinen (Klimawissenschaften, Wirtschaftswissenschaften, Umweltwissenschaften, soziale Fragen), die von der Bundesregierung für die Dauer von fünf Jahren benannt werden. Hiermit soll eine unabhängige Instanz geschaffen werden, welche mit wissenschaftlich fundierten Bewertungen und Empfehlungen den Prozess zur Erreichung der Klimaschutzziele unterstützt. ${ }^{130}$ Gemäß \$12 Abs. $1 \mathrm{KSG}$ prüft der Expertenrat die Emissionsdaten nach $\int 5$ Abs. 1 und 2 und legt der Bundesregierung und dem Deutschen Bundestag eine Bewertung der veröffentlichten Daten vor. Vor der Erstellung der Beschlussvorlage für die Bundesregierung über die Maßnahmen im Rahmen eines Sofortprogramms nach $\$ 8$ Abs. 2 KSG prüft der Expertenrat zudem die den Maßnahmen zugrunde gelegten Annahmen zur THG-Reduktion ( $\$ 12$ Abs. 2 KSG). Zudem hat die Bundesregierung vor einer Änderung der Jahresemissionsmengen durch Verordnung nach $\$ 4$ Abs. 5, der Fortschreibung des Klimaschutzplans oder dem Beschluss von Klimaschutzprogrammen eine Stellungnahme des Expertenrats einzuholen. Letztere beschränkt sich allerdings wiederum auf die diesen Entscheidungen zugrundeliegenden Annahmen zur THG-Reduktion. Im Vergleich zu den im Referentenentwurf des KSG geregelten Befugnissen wurden diese in der verabschiedeten Fassung erheblich eingeschränkt. Insbesondere ist die ursprünglich vorgesehene Bewertung der jährlichen Klimaschutzberichte der Bundesregierung entfallen (vgl. $\$ 13$ Abs. 1 S. 2 RefE). Hierdurch wird die Rolle des Expertenrates im Wesentlichen auf eine neutrale Instanz zur Überprüfung von Emissionsdaten reduziert. ${ }^{131}$

\subsubsection{Vorbildfunktion der öffentlichen Hand}

In seinem fünften Abschnitt thematisiert das KSG die Vorbildfunktion der öffentlichen Hand. Danach haben die Träger öf- fentlicher Aufgaben bei ihren Planungen und Entscheidungen den Zweck dieses Gesetzes und die zu seiner Erfüllung festgelegten Ziele zu berücksichtigen ( $\$ 13 \mathrm{KSG}$ ). Dies gilt auch im Hinblick auf Investitions- und Beschaffungsvorgänge (vgl. hierzu $\$ 13$ Abs. 2 und 3 KSG). Überall dort, wo materielles Bundesrecht Auslegungs- und Ermessenspielräume vorsieht, sind damit die Vorgaben des KSG zu berücksichtigen. Diese Verpflichtung gilt auch für die Länder und Kommunen, soweit diese Bundesrecht vollziehen. Eine strikte Beachtenspflicht besteht allerdings nicht. ${ }^{132}$ Der Bund setzt sich zudem das Ziel einer klimaneutralen Bundesverwaltung bis zum Jahr 2030 und der Verabschiedung der hierfür erforderlichen Maßnahmen (\$15 Abs. $1 \mathrm{KSG}$ ). Die Klimaneutralität der Bundesverwaltung soll insbesondere durch Energieeinsparung, Energieeffizienz und die Wahl möglichst klimaschonender Verkehrsmittel erreicht werden ( $\$ 15$ Abs. 2 KSG). Im Hinblick auf die Bund-Länder-Zusammenarbeit regelt $\$ 14 \mathrm{KSG}$, dass die Länder eigene Klimaschutzgesetze erlassen können (Öffnungsklausel). Die bestehenden Klimaschutzgesetze der Länder ${ }^{133}$ gelten unbeschadet der Vereinbarkeit mit Bundesrecht ${ }^{134}$ fort Hierdurch wird der Gefahr vorgebeugt, dass die Ländergesetze aufgrund einer abschließenden Bundesregelung nichtig werden könnten (Art. 72 Abs. 1 GG). ${ }^{135}$ Die Regelung trägt dem wichtigen Beitrag der Länder zum Erreichen der Klimaschutzziele Rechnung, der nach dem Willen des Bundesgesetzgebers künftig fortgesetzt und noch ausgebaut werden soll. ${ }^{136}$

\subsection{Bewertung}

Die Schaffung eines Bundesklimaschutzgesetzes ist sehr zu begrüßen, denn es trägt vor dem Hintergrund völkerund europarechtlicher Verpflichtungen zur Erhöhung der Rechtsverbindlichkeit und Sichtbarkeit des Klimaschutzes in Deutschland bei. Zugleich enthält das Gesetz wichtige Konkretisierungen, Durchführungsbestimmungen und sonstige Vorgaben, welche die Umsetzung der internationalen und europäischen Vorschriften fördern. ${ }^{137}$ Hierdurch werden die deutschen Bemühungen für den Klimaschutz international sichtbarer und erhalten mehr Gewicht. Die Landesklimaschutzgesetze (soweit vorhanden) regeln ebenfalls wichtige Instrumente zum Klimaschutz, können ein Bundesgesetz aber nicht ersetzen. In diesem Sinne leistet das KSG einen Beitrag, den Klimaschutz in das Mehrebenensystem der Bundesrepublik Deutschland im Sinne eines „,wohlgeordneten Rechts"138 einzubinden. Das Gesetz vermittelt zwar keine unmittelbaren Rechtswirkungen gegenüber dem Bürger und die Emissionsminderungsziele als solche sind nicht einklagbar, gleichwohl sind alle staatlichen Stellen

127) Das Programm wurde am 3. Dezember 2014 durch das Bundeskabinett beschlossen. Nähere Informationen unter BMU, Aktionsprogramm Klimaschutz; https://www.bmu.de/themen/ klima-energie/klimaschutz/nationale-klimapolitik/aktionsprogramm-klimaschutz/ (Zugriff: 16.2.2020).

128) BT-Drs. 19/14337, S. 34

129) S. oben Kap. 2.2.2.

130) BT-Drs. 19/14337, S. 35

131) Scharlau et al. (Fn. 99), 9

132) Scharlau et al. (Fn. 99), 6.

133) S. hierzu Kap. 3.1.

134) Siehe zur kompetenzrechtlichen Problematik Flaskühler, Föderale Klimaschutzgesetzgebung in Deutschland, 2018, S. $126 \mathrm{ff}$., 457 und Schlacke, in: Ewer/Ramsauer/Reese/Rubel, Umwelt, Ordnung, Recht. Festschrift für Hans-Joachim Koch, 2014 S. $429 \mathrm{ff}$.

135) Scharlau et al. (Fn. 99), 7; Sina, EurUP 2018, 323.

136) BT-Drs. 19/14337, S. 37

137) Hierzu näher Verheyen/Pabsch (Fn. 34), S. 8 ff.

138) Das Leitbild des wohlgeordneten Rechts geht auf Ines Härtel zurück; dies., Die Gesetzgebungskompetenzen des Bundes und der Länder im Lichte des wohlgeordneten Rechts, in: dies., Handbuch Föderalismus, 2012, $\$ 19$ Rdnr. 1 ff.; darauf bezugnehmend Flaskühler (Fn. 134), S. 56 f. 
daran gebunden (Art. 20 Abs. 3 GG). ${ }^{139}$ Dabei enthält das KSG v. a. Ziele und Verfahrensvorschriften und überlässt das „Wie“ der Zielerreichung in erster Linie dem Klimaschutzprogramm, welches von den zuständigen Ministerien mit Maßnahmen zu füllen ist. Regelungssystematisch handelt es sich hierbei um eine zielbezogene, d.h. finale Gesetzgebung, welche insbesondere aus dem Planungsrecht und europäischen Umweltrecht bekannt ist. ${ }^{140}$ Die Umsetzung der im Klimaschutzprogramm enthaltenen Maßnahmen erfordert die Änderung verschiedener Fachgesetze sowie weiterer Maßnahmen (z.B. finanzielle Förderung, Öffentlichkeitsarbeit, Beratung etc.). Demgemäß ist ein Instrumentarium geschaffen worden, das selbst zwar keine Maßnahmen vorgibt, aber darauf abzielt, solche Maßnahmen zu befördern und zu koordinieren. ${ }^{141}$ Dieser Ansatz erschließt sich im Hinblick auf den Querschnittscharakter des Klimaschutzrechts. Denn aufgrund der Bezüge zu anderen Rechtsgebieten und ihrer Heterogenität lassen sich die gesetzlichen Klimaschutzregelungen nicht sämtlich in ein umfassendes Klimaschutzgesetz integrieren. ${ }^{142}$ Daher erscheint die Beschränkung des KSG auf die Normierung von Klimaschutzzielen sowie Planungsinstrumenten zu ihrer Umsetzung, die Verankerung von Monitoring-Mechanismen, die Schaffung eines unabhängigen Sachverständigenrats und Vorgaben zur Vorbildwirkung der Bundesverwaltung folgerichtig. Bedauerlich ist allerdings, dass die Anpassung an den Klimawandel - im Gegensatz zu den aktuelleren Landesklima(schutz)gesetzen ${ }^{143}$ - nicht Gegenstand des KSG ist. Denn Klimaschutz und Klimaanpassung sind zwei Seiten einer Medaille, die nicht losgelöst voneinander betrachtet werden sollten. Ein Bundesklimagesetz, welches auch die Anpassung regelt, könnte auch den Aktivitäten zur Klimaanpassung ${ }^{144}$ einen gesetzli- chen Rahmen verleihen und zu einer stärkeren Verzahnung zwischen Klimaschutz und Klimaanpassung beitragen.

Open Access. Dieser Artikel wird unter der Creative Commons Namensnennung 4.0 International Lizenz veröffentlicht, welche die Nutzung, Vervielfältigung, Bearbeitung, Verbreitung und Wiedergabe in jeglichem Medium und Format erlaubt, sofern Sie den/die ursprünglichen Autor(en) und die Quelle ordnungsgemäß nennen, einen Link zur Creative Commons Lizenz beifügen und angeben, ob Änderungen vorgenommen wurden.

Die in diesem Artikel enthaltenen Bilder und sonstiges Drittmaterial unterliegen ebenfalls der genannten Creative Commons Lizenz, sofern sich aus der Abbildungslegende nichts anderes ergibt. Sofern das betreffende Material nicht unter der genannten Creative Commons Lizenz steht und die betreffende Handlung nicht nach gesetzlichen Vorschriften erlaubt ist, ist für die oben aufgeführten Weiterverwendungen des Materials die Einwilligung des jeweiligen Rechteinhabers einzuholen.

Weitere Details zur Lizenz entnehmen Sie bitte der Lizenzinformation auf http://creativecommons.org/licenses/by/4.0/deed.de.

Open access funding provided by Projekt DEAL.

139) Saurer (Fn. 5), 1574

140) Näher hierzu Albrecht, Umweltqualitätsziele im Gewässerschutzrecht, 2007, S. 30, $97 \mathrm{ff}$. m.w. N.

141) Wickel, DVB1. 2013, 77, 78.

142) Wickel (Fn. 141), 78.

143) Z.B. ThürKlimaG, Energiewendegesetz Bln, EWKG S-H, s. oben Kap. 3.1.

144) Z.B. Bundesregierung, Deutsche Anpassungsstrategie an den Klimawandel (DAS), 2008; Aktionsplan Anpassung der Deutschen Anpassungsstrategie (APA I, 2011, APA II, 2015).

\title{
UNESCO Global Geoparks: Rechtsinstrumente der Unterschutzstellung im deutschen Recht - Analyse und Empfehlungen*
}

\author{
Wolfgang Köck und Rainer Wolf
}

\begin{abstract}
(C) Der/die Autor(en) 2020
UNESCO-Geoparks sind neben den Weltnaturerbestätten und den Weltkulturerbestätten eine weitere Kategorie der internationalen Anerkennung besonders qualifizierter Stätten und Landschaften. Der Beitrag untersucht die Unterschutzstellungsoptionen für Geoparks im deutschen Recht, dabei werden unterschiedliche Rechtsinstrumente des Fachrechts und der Raumordnung betrachtet und vergleichend bewertet. Ein besonderes Augenmerk gilt der Frage, ob und ggf. unter welchen Voraussetzungen Erneuerbare Energien-Projekte Einfluss auf die Anerkennung von Geoparks haben können.
\end{abstract}

\section{Einleitung}

Im Beitrag ,Unesco Global Geoparks - Idee und Anerkennungsvoraussetzungen" (siehe NuR 2020, 295) wurde die

Prof. Dr. Rainer Wolf,

Dresden, Deutschland

Prof. Dr. Wolfgang Köck,

Lehrstuhl und Forschungsgebiet Umwelt- und Planungsrecht,

Universität Leipzig,

Leipzig, Deutschland
Idee und das Konzept der UNESCO Global Geoparks sowie die Voraussetzungen ihrer Anerkennung untersucht. Für die Anerkennung wuden die „Operativen Leitlinien der UNESCO" zugrunde gelegt.

Die Operativen Leitlinien der UNESCO sind in der Bundesrepublik kein unmittelbar geltendes Recht. Bereits ihre Rechtsqualität als Normen des Völkerrechts ist klärungsbedürftig. Anders als etwa die dem Schutz des Weltkultur- und Naturerbes dienende Welterbekonvention ${ }^{1}$ liegt ihnen kein zwischen den Staaten verbindlich vereinbartes völkerrechtliches Übereinkommen zugrunde. Das Regelwerk der OLL entspringt auch weder dem Völkergewohn-

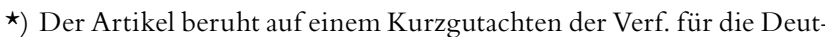
sche UNESCO-Kommission im Jahr 2018. Die Ergebnisse sind eingeflossen in ein Positionspapier des Nationalkomitees für UNESCO Global Geoparks in Deutschland v. 27.5.2019 zum Thema „Erneuerbare Energien in UNESCO-Global Geoparks“. Wir danken dem stv. Generalsekretär der Deutschen UNESCO-Kommission e. V., Dr. Lutz Möller, für eine konstruktive Zusammenarbeit.

1) Bekanntmachung des Übereinkommens zum Schutz des Kulturund Naturerbes der Welt vom 2.2. 1977, BGBl. 1977 II S. 213. 